\title{
Thrombolytic effect of Siddha herbal formulation Sikkanjar Manapagu using in-Silico model
}

\author{
Research Article
}

\section{Panneerselvam N R ${ }^{*}$, Anbarasan B2 ${ }^{2}$, Subathra T3}

\section{Professor, Department of Kuzhanthai Maruthuvam, Sri Sai Ram Siddha Medical College and Research Centre, Sai Leo Nagar, Tambaram West, Chennai. India.}

2. Assistant Professor, Department of Maruthuvam, National Institute of Siddha, Tambaram Sanatorium, Chennai, India. 3. Ph.D. Scholar, Department of Nanju Maruthuvam, National Institute of Siddha, Tambaram Sanatorium, Chennai, India.

\begin{abstract}
Background: Thromboembolic disorders are one of the important causes leading to death. In the Siddha system of medicine, many drugs have been mentioned in the literature and their thrombolytic potential needs to be scientifically evaluated. Aim: The study aims to perform the In Silico computational studies of Phytoconstituents of Siddha formulation Sikkanjar Manapagu (SM) to evaluate its thrombolytic potential. Methods: Autodock program was used for the molecular docking studies of the retrieved phytoconstituents such as Zingiberene, Gingerenone-A, 6 Gingerol of Zingiber officinale, Menthol, Luteolin, Citronellol of Mentha arvensis, Eugenol, Limonene, Myrcene, and Linalool of Citrus aurantium against target protein Human Plasminogen Activation Loop Peptide - PDB 4DCB. Results: A total of ten compounds were screened, of these Zingiberene, Menthol, Citronellol, Eugenol, Limonene, Myrcene, and Linalool showed high binding against active amino acid residue 195. Conclusion: Based on further experiments and clinical trials, the formulation Sikkanjar Manapagu could be proved to be effective in thrombolytic treatment.
\end{abstract}

Key Words: Docking study, Thrombolytic, Siddha formulation, Sikkanjar manapagu, Human Plasminogen Activation Loop Peptide - PDB 4DCB.

\section{Introduction}

A thrombus is a solid mass formed in blood vessels from the components of blood and is colloquially called a "blood clot". Thrombosis occurs due to the imbalance in normal homeostasis in the circulatory system. It is a fatal disease and it can occur from the heart, arteries, and veins or its microcirculation, named cardiac thrombi, arterial thrombi, venous thrombi, and capillary thrombi based on its origin $(1,2)$. Thrombi are comprised of platelets, fibrin, erythrocytes, leukocytes, and extracellular traps of neutrophils (3). Unnecessary formation of thrombus may lead to serious consequences like embolism, ischemia, myocardial infarction, stroke, and so on (4). The fragments of thrombi can be separated off and may be passed to various locations via blood circulation. The broken piece of thrombus in the circulation is "embolus" which may obstruct the blood vessels and the condition is called as "Embolism". These obstacles hinder the supply of oxygen to the adjacent tissue,

\section{* Corresponding Author:}

\section{Panneerselvam N R}

Professor, Department of Kuzhanthai Maruthuvam,

Sri Sai Ram Siddha Medical College and Research

Centre, Sai Leo Nagar, Tambaram West,

Chennai. India

Email Id: nrpannier@gmail.com leading to the tissue's degradation and death. Based on the situation of thrombi or emboli, they may cause various diseases such as deep vein thrombosis (caused by venous thrombi), pulmonary embolism, arterial thrombosis, stroke, acute myocardial infarction (caused by cardiac thrombi), and retinal artery occlusion (5). An important event in the dissolution of the intravascular blood clot is plasminogen activation (6). Therefore, Plasminogen Activation Loop was chosen as the target for this study. The binding of phytocomponents with the core amino acid residue 195 plays a critical role in the recognition of the residues Arg561-Va1562 of plasminogen. It has been found to have a similar pose in the mutant form. Thereby phytocomponents that bind with the amino acid 195 may be expected to medicate the cleavage of zymogen plasminogen at its Arg561Val562. Further, these leads may be considered as potential thrombolytic agents. Thrombolytics are plasminogen activators, which are the substances used in the treatment of dissolving intravascular blood clots. Thrombolytics that are currently available are, Streptokinase, Urokinase, Streptokinase, Alteplase, Reteplase, Tenecteplase, Prourokinase, Anistreplase, etc. But there are chances of the occurrence of adverse reactions such as bleeding (most common), hypotension, angioedema, etc., while using these drugs (7). Because of these drawbacks of synthetic and recombinant thrombolytic drugs, the mergence of alternatives should be appreciated and also natural 
products may be good replacements to the currently used thrombolytic drugs (8). This research aims to analyze the thrombolytic potential of Sikkanjar Manapagu using an in-silico model. The drug Sikkanjar Manapagu is a syrup-based herbal preparation, mentioned in the Siddha literature. SM has Zingiber officinale, Mentha arvensis, Citrus aurantium, Cicer arietinum, and Saccharum officinarum (9).

\section{Materials and methods \\ Ligand Preparation}

The crystalline structure of the target protein Human Plasminogen Activation Loop Peptide - PDB 4DCB (Fig.1) was retrieved from the protein data bank. The protein clean-up process was done and essential missing hydrogen atoms were being added. Different orientation of the lead molecules with respect to the target protein was evaluated by Autodock program and the best dock pose was selected based on the interaction study analysis.

\section{Methodology}

Docking calculations were carried out for retrieved phytocomponents, Zingiberene, GingerenoneA, 6 Gingerol of Zingiber officinale (10-11), Menthol, Luteolin, Citronellol of Mentha arvensis (12), Eugenol, Limonene, Myrcene, and Linalool of Citrus aurantium (13) against target protein Human Plasminogen Activation Loop Peptide - PDB 4DCB. Essential hydrogen atoms, Kollman united atom type charges, and solvation parameters were added with the aid of AutoDock tools. Affinity (grid) maps of $\times \times \AA$ grid points and $0.375 \AA$ spacing were generated using the Autogrid program. AutoDock parameter set- and distance-dependent dielectric functions were used in the calculation of the van der Waals and the electrostatic terms, respectively. Docking simulations were performed using the Lamarckian genetic algorithm (LGA) and the Solis \& Wets local search method. The initial position, orientation, and torsions of the ligand molecules were set randomly. All rotatable torsions were released during docking. Each docking experiment was derived from 2 different runs that were set to terminate after a maximum of 250000 energy evaluations. The population size was set to 150 . During the search, a translational step of $0.2 \AA$, and quaternion and torsion steps of 5 were applied (14-18).

Fig.1: 3D- Structure of Human Plasminogen Activation Loop Peptide - PDB 4DCB

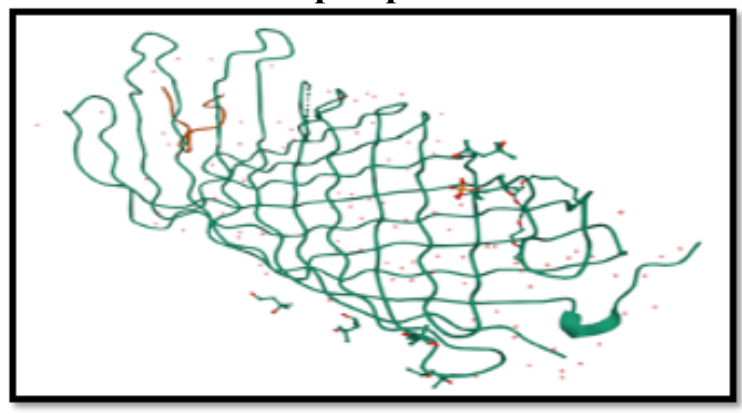

Fig.2: 2D and 3D Structure of Selected Ligands

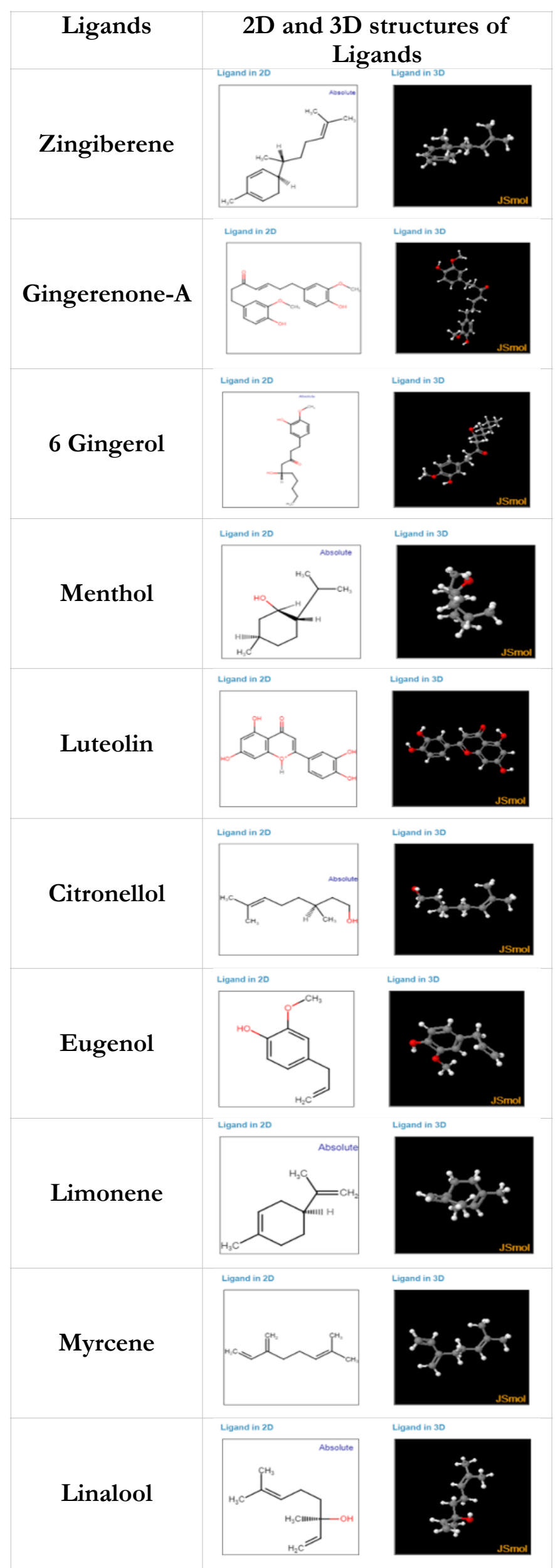


International Journal of Ayurvedic Medicine, Vol 12 (4), 908-911

Table 1: Ligand Properties of the Compounds Selected for Docking Analysis

\begin{tabular}{|c|c|c|c|c|c|}
\hline Compound & Molar weight g/mol & Molecular Formula & H Bond Donor & $\begin{array}{c}\text { H Bond } \\
\text { Acceptor }\end{array}$ & $\begin{array}{c}\text { Rotatable } \\
\text { bonds }\end{array}$ \\
\hline Zingiberene & $204.35 \mathrm{~g} / \mathrm{mol}$ & $\mathrm{C}_{15} \mathrm{H}_{24}$ & 0 & 0 & 4 \\
\hline Gingerenone-A & $356.4 \mathrm{~g} / \mathrm{mol}$ & $\mathrm{C}_{21} \mathrm{H}_{24} \mathrm{O}_{5}$ & 2 & 5 & 9 \\
\hline 6 Gingerol & $294.391 \mathrm{~g} / \mathrm{mol}$ & $\mathrm{C}_{17} \mathrm{H}_{26} \mathrm{O}_{4}$ & 2 & 4 & 10 \\
\hline Menthol & $156.26 \mathrm{~g} / \mathrm{mol}$ & $\mathrm{C}_{10} \mathrm{H}_{20} \mathrm{O}$ & 1 & 1 & 1 \\
\hline Luteolin & $286.24 \mathrm{~g} / \mathrm{mol}$ & $\mathrm{C}_{15} \mathrm{H}_{10} \mathrm{O}_{6}$ & 4 & 6 & 1 \\
\hline Citronellol & $156.26 \mathrm{~g} / \mathrm{mol}$ & $\mathrm{C}_{10} \mathrm{H}_{20} \mathrm{O}$ & 1 & 1 & 5 \\
\hline Eugenol & $164.2 \mathrm{~g} / \mathrm{mol}$ & $\mathrm{C}_{10} \mathrm{H}_{12} \mathrm{O}_{2}$ & 1 & 2 & 3 \\
\hline Limonene & $136.23 \mathrm{~g} / \mathrm{mol}$ & $\mathrm{C}_{10} \mathrm{H}_{16}$ & 0 & 0 & 1 \\
\hline Myrcene & $136.238 \mathrm{~g} / \mathrm{mol}$ & $\mathrm{C}_{10} \mathrm{H}_{16}$ & 0 & 0 & 4 \\
\hline Linalool & $154.25 \mathrm{~g} / \mathrm{mol}$ & $\mathrm{C}_{10} \mathrm{H}_{18} \mathrm{O}$ & 1 & 1 & 4 \\
\hline
\end{tabular}

Table 2: Summary of the molecular docking studies of compounds against Human Plasminogen Activation Loop Peptide - PDB 4DCB

\begin{tabular}{|c|c|c|c|c|c|}
\hline Compounds & $\begin{array}{c}\text { Binding Free } \\
\text { energy Kcal/ } \\
\text { mol }\end{array}$ & $\begin{array}{c}\text { Inhibition constant } \\
\text { Ki } \boldsymbol{\mu M}(* \mathbf{M M}) \\
(* * \mathbf{n M})\end{array}$ & $\begin{array}{c}\text { Electrostatic } \\
\text { energy Kcal/mol }\end{array}$ & $\begin{array}{c}\text { Intermolecular } \\
\text { energy Kcal/mol }\end{array}$ & $\begin{array}{c}\text { Total } \\
\text { Interaction } \\
\text { Surface }\end{array}$ \\
\hline Zingiberene & -5.86 & 50.77 & -0.03 & -6.92 & 527.87 \\
\hline Gingerenone-A & -6.07 & 35.35 & -0.17 & -7.33 & 623.33 \\
\hline 6 Gingerol & -4.32 & 685.12 & -0.10 & -6.16 & 554.34 \\
\hline Menthol & -4.76 & 326.58 & -0.24 & -5.33 & 365.97 \\
\hline Luteolin & -5.28 & $135.69 *$ & -0.34 & -4.97 & 470.09 \\
\hline Citronellol & -4.81 & 297.00 & -0.14 & -6.49 & 410.22 \\
\hline Eugenol & -4.98 & 223.32 & -0.23 & -5.35 & 383.88 \\
\hline Limonene & -4.80 & 302.96 & -0.04 & -5.10 & 357.68 \\
\hline Myrcene & -4.89 & 261.69 & -0.01 & -6.02 & 402.24 \\
\hline Linalool & -5.26 & 138.51 & -0.29 & -6.67 & 403.70 \\
\hline
\end{tabular}

Table 3: Amino acid Residue Interaction of Lead against Human Plasminogen Activation Loop Peptide - PDB 4DCB

\begin{tabular}{|c|c|c|c|c|c|c|c|c|c|c|c|c|c|}
\hline Molecule & Interactions & \multicolumn{12}{|c|}{ Amino Acid Residue- Binding } \\
\hline Zingiberene & 1 & 19 SER & $\begin{array}{c}54 \\
\text { LYS }\end{array}$ & $\begin{array}{c}56 \\
\text { ASP }\end{array}$ & $\begin{array}{c}70 \\
\text { ARG }\end{array}$ & $\begin{array}{c}72 \\
\text { TRP }\end{array}$ & $\begin{array}{c}111 \\
\text { GLU }\end{array}$ & $\begin{array}{c}113 \\
\text { ASP }\end{array}$ & $\begin{array}{c}195 \\
\text { LYS }\end{array}$ & $\begin{array}{c}244 \\
\text { GLU }\end{array}$ & $\begin{array}{c}246 \\
\text { THR }\end{array}$ & $\begin{array}{c}248 \\
\text { SER }\end{array}$ & \\
\hline Gingerenone-A & 0 & 179 LEU & $\begin{array}{c}194 \\
\text { PHE }\end{array}$ & $\begin{array}{c}196 \\
\text { PHE }\end{array}$ & $\begin{array}{c}225 \\
\text { TYR }\end{array}$ & $\begin{array}{l}249 \\
\text { LYS }\end{array}$ & $\begin{array}{l}251 \\
\text { ASP }\end{array}$ & & & & & & \\
\hline 6 Gingerol & 0 & 179 LEU & $\begin{array}{c}194 \\
\text { PHE }\end{array}$ & $\begin{array}{c}196 \\
\text { PHE }\end{array}$ & $\begin{array}{c}223 \\
\text { ARG }\end{array}$ & $\begin{array}{c}225 \\
\text { TYR }\end{array}$ & $\begin{array}{l}251 \\
\text { ASP }\end{array}$ & & & & & & \\
\hline Menthol & 1 & $56 \mathrm{ASP}$ & $\begin{array}{c}68 \\
\text { ASP }\end{array}$ & $\begin{array}{c}70 \\
\text { ARG }\end{array}$ & $\begin{array}{c}113 \\
\text { ASP }\end{array}$ & $\begin{array}{l}115 \\
\text { ASP }\end{array}$ & $\begin{array}{c}117 \\
\text { LYS }\end{array}$ & $\begin{array}{c}193 \\
\text { LEU }\end{array}$ & $\begin{array}{c}195 \\
\text { LYS }\end{array}$ & $\begin{array}{c}228 \\
\text { VAL }\end{array}$ & $\begin{array}{c}230 \\
\text { ASN }\end{array}$ & & \\
\hline Luteolin & 0 & 179 LEU & $\begin{array}{c}196 \\
\text { PHE }\end{array}$ & $\begin{array}{c}223 \\
\text { ARG }\end{array}$ & $\begin{array}{c}225 \\
\text { TYR }\end{array}$ & $\begin{array}{l}251 \\
\text { ASP }\end{array}$ & & & & & & & \\
\hline Citronellol & 1 & 54 LYS & $\begin{array}{c}70 \\
\text { ARG }\end{array}$ & $\begin{array}{c}72 \\
\text { TRP }\end{array}$ & $\begin{array}{c}111 \\
\text { GLU }\end{array}$ & $\begin{array}{l}113 \\
\text { ASP }\end{array}$ & $\begin{array}{c}195 \\
\text { LYS }\end{array}$ & $\begin{array}{c}224 \\
\text { TYR }\end{array}$ & $\begin{array}{r}228 \\
\text { VAL }\end{array}$ & $\begin{array}{c}246 \\
\text { THR }\end{array}$ & $\begin{array}{c}248 \\
\text { SER }\end{array}$ & $\begin{array}{c}281 \\
\text { ASN }\end{array}$ & \\
\hline Eugenol & 1 & 54 LYS & $\begin{array}{c}70 \\
\text { ARG }\end{array}$ & $\begin{array}{c}72 \\
\text { TRP }\end{array}$ & $\begin{array}{c}111 \\
\text { GLU }\end{array}$ & $\begin{array}{l}113 \\
\text { ASP }\end{array}$ & $\begin{array}{c}195 \\
\text { LYS }\end{array}$ & $\begin{array}{c}224 \\
\text { TYR }\end{array}$ & $\begin{array}{r}228 \\
\text { VAL }\end{array}$ & $\begin{array}{l}248 \\
\text { SER }\end{array}$ & $\begin{array}{c}281 \\
\text { ASN }\end{array}$ & & \\
\hline Limonene & 1 & $54 \mathrm{LY}$ & $\begin{array}{c}70 \\
\text { ARG }\end{array}$ & $\begin{array}{c}72 \\
\text { TRP }\end{array}$ & $\begin{array}{c}111 \\
\text { GLU }\end{array}$ & $\begin{array}{c}113 \\
\text { ASP }\end{array}$ & $\begin{array}{l}195 \\
\text { LYS }\end{array}$ & $\begin{array}{c}224 \\
\text { TYR }\end{array}$ & $\begin{array}{c}228 \\
\text { VAL }\end{array}$ & $\begin{array}{c}246 \\
\text { THR }\end{array}$ & $\begin{array}{l}248 \\
\text { SER }\end{array}$ & & \\
\hline Myrcene & 1 & 54 LYS & $\begin{array}{c}70 \\
\text { ARG }\end{array}$ & $\begin{array}{c}72 \\
\text { TRP }\end{array}$ & $\begin{array}{c}111 \\
\text { GLU }\end{array}$ & $\begin{array}{l}113 \\
\text { ASP }\end{array}$ & $\begin{array}{c}195 \\
\text { LYS }\end{array}$ & $\begin{array}{c}224 \\
\text { TYR }\end{array}$ & $\begin{array}{r}228 \\
\text { VAL }\end{array}$ & $\begin{array}{c}246 \\
\text { THR }\end{array}$ & $\begin{array}{c}248 \\
\text { SER }\end{array}$ & & \\
\hline Linalool & 1 & 54 LYS & $\begin{array}{c}70 \\
\text { ARG }\end{array}$ & $\begin{array}{c}72 \\
\text { TRP }\end{array}$ & $\begin{array}{c}111 \\
\text { GLU }\end{array}$ & $\begin{array}{l}113 \\
\text { ASP }\end{array}$ & $\begin{array}{c}135 \\
\text { GLN }\end{array}$ & $\begin{array}{c}195 \\
\text { LYS }\end{array}$ & $\begin{array}{c}224 \\
\text { TYR }\end{array}$ & $\begin{array}{c}228 \\
\text { VAL }\end{array}$ & $\begin{array}{c}246 \\
\text { THR }\end{array}$ & $\begin{array}{c}248 \\
\text { SER }\end{array}$ & $\begin{array}{l}281 \\
\text { ASN }\end{array}$ \\
\hline
\end{tabular}

\section{Results and discussion}

Thrombolytic treatment plays a key role in the management of cardiovascular diseases. A total of ten bioactive lead compounds were retrieved from the herbs present in the formulation and they are known to be Zingiberene, Gingerenone-A, 6 Gingerol of Zingiber officinale, Menthol, Luteolin, Citronellol of Mentha arvensis, Eugenol, Limonene, Myrcene, and Linalool of Citrus aurantium. From reported data of the herb, the leads like Zingiberene, Menthol, Citronellol, Eugenol, Limonene, Myrcene, and Linalool bound with active amino acid residue 195 that plays a critical role in the recognition of the residues Arg561-Val562 of target plasminogen.

While comparing binding affinities (Table 2) of the Phyto compounds, it was found that Zingiberene showed the highest binding affinity of $-5.86 \mathrm{Kcal} / \mathrm{mol}$ to amino acid residue 195. Linalool showed the secondhighest binding affinity with the binding free energy 
of-5.26Kcal $/ \mathrm{mol}$, followed by Eugenol, Myrcene, Citronellol, and limonene with binding energies -4.98 $\mathrm{Kcal} / \mathrm{mol},-4.89 \mathrm{Kcal} / \mathrm{mol},-4.81 \mathrm{Kcal} / \mathrm{mol}$, and -4.80 $\mathrm{Kcal} / \mathrm{mol}$ respectively. Menthol has the lowest binding affinity $(-4.76 \mathrm{Kcal} / \mathrm{mol})$ to amino acid residue 195 . The compounds Zingiberene, Linalool, Eugenol, Myrcene, Citronellol, Limonene, and Menthol shared one active site amino acid in common (Table 3). Though the compound Gingerenone-A has the highest binding energy among all the compounds, while considering the interactions, it did not show any interactions with the amino acid residue 195. Similarly, 6 Gingerol and Luteolin did not show any interactions with the amino acid residue 195. As a whole, considering its bonding affinity and number of interactions with the amino acid residue 195, seven possible inhibitors were identified against the target protein Human Plasminogen Activation Loop Peptide - PDB 4DCB.

\section{Conclusion}

Based on the results of the computational analysis, it was concluded that the bioactive compounds like Zingiberene, Menthol, Citronellol, Eugenol, Limonene, Myrcene and Linalool present in Zingiber officinale, Mentha arvensis, and Citrus aurantium of Sikkanjar Manapagu reveals significant binding against target plasminogen. Thereby it was concluded that these compounds may exert promising anti-thrombolytic activity.

\section{Conflict of interest} Nil

\section{Reference}

1. Harsh Mohan. Textbook of Pathology. 8ed. New Delhi. Jaypee brothers medical publishers; 2019. 183-186p.

2. Mahmud S, Akhter S, Rahman M.D, Aklima J, Akhter S, Merry S.R, Jubair S.M, Dash R, Emran T Bin, Antithrombotic effects of five organic extracts of Bangladeshi plants in vitro and mechanisms in in silico models. Evid.-Based Complement. Altern. Med. May, 2015.

3. Ghadir Alkarithi, Cédric Duval, Yu Shi, Fraser L. Macrae, Robert A.S. Ariëns, Thrombus Structural Composition in Cardiovascular Disease. Arteriosclerosis Thrombosis and Vascular Biology. July, 2021; 41(9): 2370-2383.

4. Ali MR, Salim Hossain M, Islam MA, Saiful Islam Arman M, Sarwar Raju G, Dasgupta P, Noshin TF. Aspect of thrombolytic therapy: a review. Scientific World Journal. December, 2014;2014

5. Adivitiya, Khasa YP. The evolution of recombinant thrombolytics: Current status and future directions. Bioengineered. July 2017; 4;8(4);331-358.
6. Marina A.A Parry, Xuejun C Zhang, Wolfram Bode, Molecular mechanisms of plasminogen activation: bacterial cofactors provide clues, Trends in Biochemical Sciences. February, 2000; 25(2); 53-59

7. https://www.ncbi.nlm.nih.gov/books/NBK557411/ Thrombolytic Therapy dated June, 17, 2021

8. Islam MA, Alam F, Khalil MI, Sasongko TH, Gan SH. Natural Products Towards the Discovery of Potential Future Antithrombotic Drugs. Curr Pharm Des. 2016; 22(20); 2926-46

9. Kannusami pillai C. Chikitsa ratna deepam Irandam Baagamaagiya Vaithiya Chinthamani. 1st edition. Chennai; B. Rathina Nayagar and Sons; 1927. 253-254p.

10. Sahdeo Prasad. Ginger and Its Constituents: Role in Prevention and Treatment of Gastrointestinal Cancer. Gastroenterology Research and Practice. February, 2015; 1-11

11. Rampogu S, Baek A, Gajula RG, et al. Ginger (Zingiber officinale) phytochemicals-gingerenoneA and shogaol inhibit SaHPPK: molecular docking, molecular dynamics simulations and in vitro approaches. Ann Clin. Microbiol. Antimicrob. April, 2018;17(1);16

12. Nazima Ameen. Pharmacological review on Mentha arvensis. International Journal of Current Research. July, 2016; 8(7); 34120-34123

13. Mohammad K. Okla. Yield, Phytochemical Constituents, and Antibacterial Activity of Essential Oils from the Leaves/Twigs, Branches, Branch Wood, and Branch Bark of Sour Orange (Citrus aurantium L.). June, 2019, 7(6); 363

14. Bikadi, Z., Hazai, E.Application of the PM6 semiempirical method to modeling proteins enhances docking accuracy of AutoDock. J. Cheminf. September, 2009; 1; 15

15. T. A. Halgren. Merck molecular force field. I. Basis, form, scope, parametrization, and performance of MMFF94. Journal of Computational Chemistry. April 1996, 17 (5-6); 490-519

16. Morris GM, Goodsell DS, et al. Automated docking using a Lamarckian genetic algorithm and an empirical binding free energy function. Journal of Computational Chemistry. 1998; 19 (14); 1639-1662

17. Solis FJ and Wets RJB. Minimization by Random Search Techniques. Mathematics of Operations Research. February, 1981; 6(1); 19-30

18. Amiri, M., Jalali-Javaran, M., Haddad, R. et al. In silico and in vivo analyses of the mutated human tissue plasminogen activator (mtPA) and the antithetical effects of P19 silencing suppressor on its expression in two Nicotiana species. Sci Rep 8. September, 2018; 8(1); 14079 